\title{
Time out from a fixed ratio schedule'
}

JOHN R. THOMAS AND JAMES A. SHERMAN

INSTITUTE FOR BEHAVIORAL RESEARCH, SILVER SPRING, MARYLAND

\begin{abstract}
Abstraet
The present study is a replication of the experimental procedure employed by Thompson (1965) to investigate the FR-TO effect. The results only partially replicate previous results showing an increasing monotonic relation between $\mathrm{TO}$ frequency and $\mathrm{FR}$ value and do not replicate the finding that TOs are restricted to the pre-ratio pause.
\end{abstract}

\section{Problem}

Several studies have reported that an organism performing on a fixed ratio (FR) schedule will occasionally respond on a second manipulandum to impose a period of time out (TO) from the FR schedule (Azrin, 1961; Appel, 1963; Thompson, 1964, 1965; Zimmerman \& Ferster, 1964). It has been suggested that the TO behavior is maintained by escape from some aversive aspect of the FR schedule (Azrin,1961; Thompson, 1964, 1965). The suggestion has been supported by evidence that the TOs typically are restricted to the pre-ratio pause of the FR schedule and that the frequency and duration of imposed TOs are increasing monotonic functions of the FR value.

The present study is essentially a replication of the fixed duration TO procedure used by Thompson (1965) to investigate the FR-TO effect.

\section{Method}

The Ss were three adult male homing pigeons maintained at $80 \%$ of free feeding weight. The apparatus was a standard two key pigeon chamber with associated automatic programming and recording equipment. The Ss were first exposed to a fixed ratio requirement of 50 responses (FR 50) on the right response key which was illuminated with a green key light. A reinforcement consisted of a 4-sec. presentation of the food tray. Following a number of sessions on the FR, the left response key was made available and illuminated with a white key light concurrently with the FR schedule. A single response on the left key extinguished both key lights and turned on the houselight for $30 \mathrm{sec}$. (TO period). Responses on either key during the 30-sec. TO period had no programmed consequence. Each session was preceded by a blackout condition during which all lights were extinguished. A session ended with a reinstatement of the blackout following $2 \mathrm{hr}$. or 50 reinforcements, whichever occurred first.

The FR requirement was increased in steps of 50 from FR 50 to FR 200. Sessions were run under each FR value until stable behavioral performances were obtained. Following the FR 150 requirement, several sessions were run during which the FR behavior was extinguished. The FR 150 requirement was reinstated and stable behavioral performances were obtained before the requirement was increased to FR 200 .

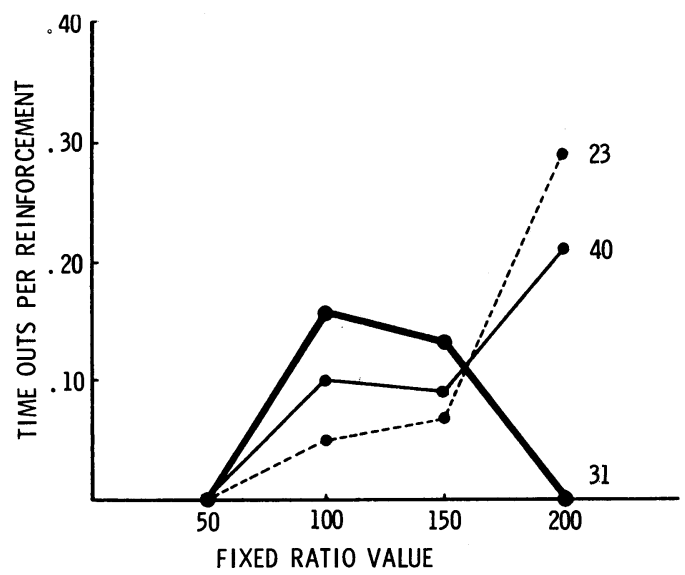

Fig. 1. Number of time out responses per FR reinforcement as a function of $F R$ value for three Ss.

\section{Results and Diseussion}

The mean number of TO responses per FR reinforcement, based on the last three sessions at each FR value, for the three Ss are presented in Fig. 1. For two Ss (23 and 40), the number of TO responses per reinforcement in general increased with increases in the size of the FR value. For the third $S(31)$, the number of TOs first increased and then decreased with increases in the FR size. The number of TO responses increased for the three Ss during the first session following an increase in ratio size and then decreased by the second or third session on the new FR value. The increase in the number of TOs with increases in FR value for the two Ss is consistent with previous studies (Azrin, 1961; Appel, 1963; Thompson, 1964, 1965). The third $\mathrm{S}$ that showed no monotonic relationship between ratio size and number of TO responses is more similar to the performances reported by Zimmerman \& Ferster (1964). It is not clear as to why the function was not replicated with the third S. The third S's ratio behavior was extremely weak at the FR 200 value. Zimmerman and Ferster reported a lower tendency for TO responses when FR behavior became weak during high ratio values. The weak FR behavior may account for the loss of TO behavior by S 31 .

The most consistent difference between previous studies and the present study is that the TO responses were not always restricted to the pre-ratio pause. At FR 100 and FR 150 the TO responses typically occurred during the pre-ratio pause, although TO responses occasionally occurred during the ratio run. At FR 200, when the number of TOs per reinforcement markedly increased for Ss 23 and 40, the majority of the TOs 

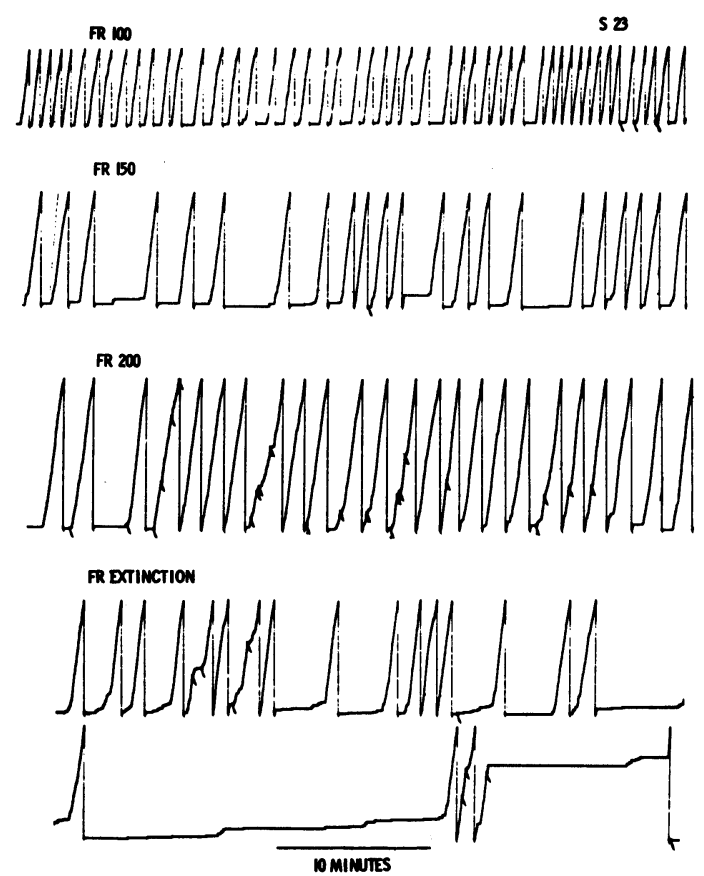

Fig. 2. Cumulative response records of $\mathbf{S} 23$ for three $\mathbf{F R}$ values. The bottom two rows show extinction performance of the FR 150.

occurred during the ratio run. In the top three rows of Fig. 2 are presented cumulative response records of performances of S 23 at three of the FR values. The recording pen resets to the baseline upon the occurrence of the food reinforcement on the FR schedule. The pips indicate TO responses. The recording paper drive was disconnected during the 30-sec. TO period. During extinction of the FR behavior TO responses initially increased and then decreased as extinction continued. The increased number of TO responses that appeared during extinction was located during the ratio run. A segment of the first extinction session of S 23 is presented in the two bottom rows of Fig. 2.

The occurrence of TOs during the ratio run at the larger FR values and during initial extinction sessions does not support the suggestion that the pre-ratio pause is the most aversive point within the FR schedule (Thompson, 1965). The present experimental procedure does not conclusively allow the interpretation of the TO responses as maintained by the removal of the $S$ from the aversive aspects of the FR schedule. Previous studies have demonstrated that stimulus change, per se, may have much to do with the control over the occurrence of TO responses in the present procedure (Appel, 1963; Zimmerman \& Ferster, 1964). It is possible that the TO behavior could occur whenever there is a momentary loss of control by the FR schedule. Temporary loss of control by the schedule of food reinforcement which allows the occurrence of the relatively weaker TO behavior has been suggested by Zimmerman \& Ferster (1964).

\section{References}

Appel, J. B. Aversive aspects of a schedule of positive reinforcement. J. exp. Anal. Behav., 1963, 6, 423-428.

Azrin, N. H. Time out from positive reinforcement. Science, 1961, $133,382-383$.

Thompson, D. M. Escape from $\mathbf{S}^{\mathrm{D}}$ associated with fixed-ratio reinforcement. J. exp. Anal. Behav., 1964, 7, 1-8.

Thompson, D. M. Time-out from fixed-ratio reinforcement: a systematic replication. Psychon. Sci., 1965, 2, 109-110.

Zimmerman, J., \& Ferster, C. B. Some notes on time out from reinforcement. J. exp. Anal. Behav., 1964, 7, 13-19.

\section{Note}

1. This research was supported by grant NsG-450, National Aeronautical and Space Administration. 